\title{
Deeply Virtual Compton Scattering and Diffractive High $t$ Photons at H1
}

\author{
Matthew Beckingham ${ }^{* *}$ \\ DESY, Notkestrasse 85, D-22607 Hamburg, Germany. \\ E-mail: matthew. beckinghamedesy.de
}

\begin{abstract}
A new preliminary $\mathrm{H} 1$ measurement of the DVCS cross section, measured during the HERA II running period, and the preliminary $\mathrm{H} 1$ measurement of diffractive high $t$ photon production are presented. The DVCS cross sections, measured in the kinematic range $6.5<Q^{2}<80 \mathrm{GeV}^{2}$, $30<W<80 \mathrm{GeV}$ and $|t|<1 \mathrm{GeV}^{2}$ as a function of $t, Q^{2}$ and $W$, are in good agreement with the previously published $\mathrm{H} 1$ results and are compatible with the ZEUS measurement. Fits to the combined H1 results of the form $\exp (-b|t|),\left(1 / Q^{2}\right)^{n}$ and $W^{\delta}$ are able to describe the $t, Q^{2}$ and $W$ cross sections, respectively. The data are described by NLO QCD and colour dipole model predictions in both shape and normalisation.

Diffractive high $t$ photon production cross sections have been measured differentially as a function of $x_{\mathbb{P}}$ and $t$, in the kinematic range $p_{t(\gamma)}>2 \mathrm{GeV}, 175<W<247 \mathrm{GeV}, 1 \times 10^{-4}<x_{\mathbb{P}}<7 \times 10^{-4}$ and $y_{\mathbb{P}}<0.018$. The cross sections are found to have a reasonable agreement in both normalisation and shape with a LLA BFKL prediction. In particular, the cross section is found to rise very steeply with decreasing $x_{\mathbb{P}}$.
\end{abstract}

DIFFRACTION 2006 - International Workshop on Diffraction in High-Energy Physics September 5-10 2006

Adamantas, Milos island, Greece

*Speaker.

$\dagger$ On behalf of the H1 Collaboration 


\section{Introduction}

Deeply Virtual Compton Scattering (DVCS), the process $e p \rightarrow e \gamma p$, and Diffractive High $t$ Photon Production, $e p \rightarrow e \gamma Y$ where the photon carries a large transverse momentum and is well separated from the proton dissociative system $Y$, are processes with similar final states - both consist of a final state electron and photon well separated from the final state proton system. However, they lie in different kinematic regimes - high $Q^{2}$ and low $|t|$ for DVCS, but low $Q^{2}$ and high $|t|$ for high $t$ photons. Both processes provide clean probes of diffractive processes. They are experimentally clean - DVCS and high $t$ photon production rely on the measurement of the final state electron and photon, with the additional requirement of the proton system being forward. In addition, both processes are theoretically favorable - with the hard scale present in both processes $\left(Q^{2}\right.$ in DVCS and $t$ in high $t$ photons), perturbative QCD calculations may be made that are not plagued by the lack of understanding of the meson wavefunction that enters calculations of vector meson electroproduction. The only non-perturbative contribution to the cross section comes from the structure of the proton.

This paper presents a new preliminary measurement of DVCS alongside the preliminary measurement of Diffractive High $t$ Photon production. More details may be found in [1] and [2].

\section{H1 Detector}

A detailed description of the $\mathrm{H} 1$ detector can be found elsewhere [3]. The following briefly describes the detector components relevant to the two analyses presented here. The Liquid Argon (LAr) calorimeter covers the polar angle range $4^{\circ}<\theta<153^{\circ}$ with full azimuthal coverage ${ }^{1}$. The angular region $154^{\circ}<\theta<174^{\circ}$ is covered by the lead-scintillating fibre SpaCal calorimeter. Both calorimeters contain electromagnetic and hadronic sections. Tracking coverage is provided in the polar range $20^{\circ}<\theta<160^{\circ}$ by the central track detector. Momentum measurements are possible by the $1.15 \mathrm{~T}$ magnetic field, uniform over the full tracker volume, provided by the solenoid that surrounds the calorimeter volume. Identification of electrons in the $\mathrm{SpaCal}$ and measurement of their angle is aided by the Backwards Proportional Chamber (BPC), situated in front of the SpaCal. The Forward Muon Detector (FMD) is used to tag hadronic activity in the polar range $3^{\circ}<\theta<17^{\circ}$, occurring in proton dissociation events where the secondary particles interact with the beam pipe. The luminosity is measured using a brace of crystal calorimeters situated near to the electron beam pipe in the outgoing electron direction. In addition, one of the two detectors is used to measure the final state electron when scattered through a small angle.

\section{DVCS}

The leading order diagram for DVCS at HERA and one contribution to the next-to-leading order are shown in figure 1. The transition of the virtual photon to a real photon forces the fractional momenta of the two exchanged partons to be different or "skewed". Hence, DVCS is sensitive to the correlations between partons in the proton encoded in the Generalised Parton Distributions

\footnotetext{
${ }^{1} \mathrm{~A}$ right handed coordinate is defined with the $z$, or forward, direction defined in the direction of the outgoing proton beam.
} 


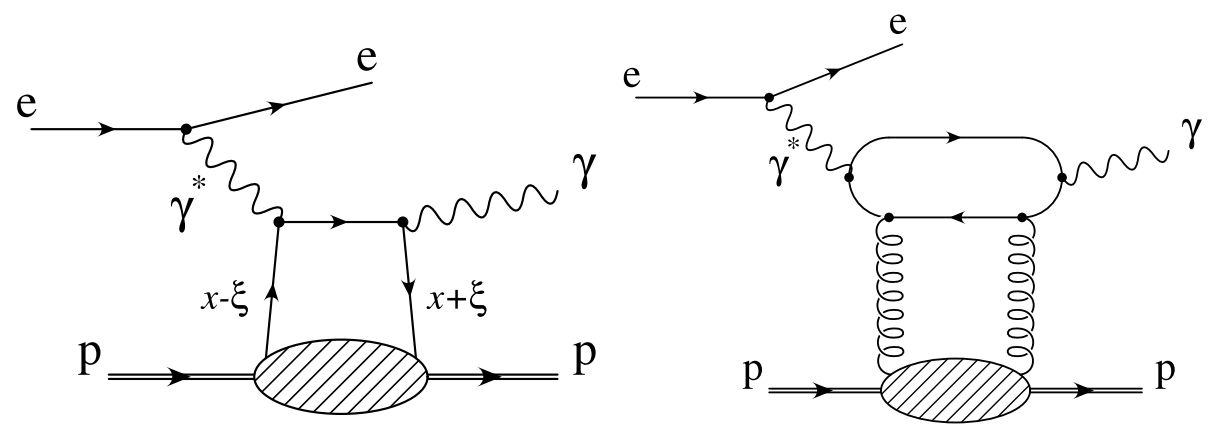

Figure 1: Diagrams contributing to the DVCS process at (a) leading order and (b) next-to-leading order.

(GPDs). The GPDs generalise and interpolate between the usual proton PDFs and elastic form factors - whereas PDFs contain information on the longitudinal momenta of the parton within the proton, elastic form factors contain information on the partons transverse momenta. In light cone coordinates, GPDs represent the interference between two different parton wavefunctions, one with momenta $x+\xi$ and the other with momenta $x-\xi$, as shown in figure 1. Here the variable $\xi$ represents the skewedness of the exchanged partons. The GPDs also depend on $x$ and the four momentum transfer at the proton vertex squared $t$. GPDs are defined at a starting scale $\mu^{2}$ and their $Q^{2}$ evolution is governed by pQCD.

In the following, the DVCS data are compared to NLO QCD predictions for the DVCS cross section and the prediction from a colour dipole model. The NLO QCD predictions are obtained from Freund and McDermott [4, 5] using two different GPD parametrisations [6]. In the DGLAP region, $|x|>\xi$, the proton PDFs at a starting scale $\mu^{2}$ from MRST2001 and CTEQ6 are used to parametrise the GPDs $H^{q}\left(x, \xi, t, \mu^{2}\right)$, with the $t$ dependence factorised as an exponential behaviour. In the ERBL region, $|x|<\xi$, the quark singlet and gluon distributions are parametrised as simple analytic functions which satisfy continuation to the DGLAP region. The $Q^{2}$ and $\xi$ dependences are generated dynamically using evolution equations and no intrinsic skewedness is included at the starting scale.

Colour dipole models are based on a time factorisation of the DVCS process, viewed in the proton rest frame as three subprocesses at well separated time scales. Firstly the incoming virtual photon fluctuates into a $q \bar{q}$ pair, which then interacts with the proton and finally annihilates into the final state photon. The first and third steps are described by photon QED wavefunctions. The interaction between the $q \bar{q}$ dipole and the proton is described by the colour dipole cross section $\sigma_{\text {dipole }}\left(x, Q^{2}, r^{2}\right)$, where $r$ represents the transverse size of the dipole. The cross section $\sigma_{\text {dipole }}$ is parametrised in colour dipole predictions and an exponential form for the $t$ slope assumed. The data in this analysis are compared to a colour dipole model from Favart and Machado [7, 8]. This model applies to DVCS the saturation model of Golec-Biernat and Wüsthoff [9], used to describe diffractive DIS, combined with a DGLAP evolution for $\sigma_{\text {dipole }}$ by Bartels, Golec-Biernat and Kowalski (BGBK) [10].

Interference to the DVCS process comes from the Bethe-Heitler (BH) process $e p \rightarrow e \gamma p$. This purely electromagnetic process is precisely calculable within QED using elastic form factors and, since the interference term vanishes when integrating over azimuthal angles, this background may 
be subtracted to give the DVCS cross section. In addition, the precise knowledge of this background is useful, as the process may also be used to study the detector response.

\subsection{Data Analysis}

The preliminary measurement of DVCS is based on an integrated luminosity of $39.7 \mathrm{pb}^{-1}$ collected by the $\mathrm{H} 1$ detector in the year 2004 during the HERA II running period. During this time HERA collided positrons with an energy of $27.6 \mathrm{GeV}$ with protons of energy $920 \mathrm{GeV}$. More details of the analysis may be found in [1].

The analysis is based on requiring two electromagnetically interacting particles within the $\mathrm{H} 1$ detector. To enhance the ratio of DVCS to BH events the photon, identified by an electromagnetic energy shower in the LAr calorimeter with no associated track from the CTD, is required to be detected in the forward or central region of the $\mathrm{H} 1$ detector and have a transverse momenta $p_{T}>2 \mathrm{GeV}$. The scattered positron is identified as an electromagnetic cluster in the SpaCal with an energy $E>20 \mathrm{GeV}$, with the BPC used to determine the positron scattering angle and position in the SpaCal. Elastic events are selected and the proton dissociative background suppressed by requiring no further energy deposits above the noise threshold in the LAr calorimeter and the two layers of the FMD closest to the interaction point. The effect of QED radiative corrections are reduced by requiring the longitudinal momentum balance $\sum\left(E-p_{z}\right)>45 \mathrm{GeV}$. The events satisfying these conditions within the phase space $6.5<Q^{2}<80 \mathrm{GeV},|t|<1 \mathrm{GeV}^{2}$ and $30<W<140 \mathrm{GeV}$ form the DVCS enriched sample. To study the detector response a $\mathrm{BH}$ control sample is made. This sample is found with the same selection criteria as the DVCS enriched sample, but with the photon replacing the positron and vice versa - that is, the positron is required to be found in the forward or central region of the LAr calorimeter with a $p_{T}>2 \mathrm{GeV}$ and the photon should be found backwards in the SpaCal with an energy $E>20 \mathrm{GeV}$.

The kinematic quantities $Q^{2}, x$ and $W$ are reconstructed using the double-angle method. The four-momentum transfer squared at the proton vertex is reconstructed using the transverse momentum of the final state positron and photon, $p_{T}^{e}$ and $p_{T}^{\gamma}$ respectively, as $t \simeq-\left(p_{T}^{e}+p_{T}^{\gamma}\right)^{2}$.

\subsection{Monte Carlo Simulations and Data Corrections}

Monte Carlo simulations are used to correct the data for detector acceptance and resolution effects and for initial state radiation. Elastic and dissociative DVCS is generated using the MILOU generator [11], based on the NLO QCD DVCS prediction from Freund and McDermott. The elastic and dissociative events are generated with $|t|$ slopes of $b=6.5 \mathrm{GeV}^{-2}$ and $b=1.5 \mathrm{GeV}^{-2}$, respectively. The Compton 2.20 [12] generator, interfaced to SOPHIA [13], is used to generate both elastic and dissociative $\mathrm{BH}$ events. The backgrounds from elastic diffractive $\rho$ production and dilepton production in two photon exchange are taken into account using the DIFFVM [14] and GRAPE [15] generators, respectively. All generated events are passed through a detailed simulation of the $\mathrm{H} 1$ detector and are subject to the same reconstruction and analysis chain as the data. All MC predictions are normalised to the luminosity of the data.

A full systematic error analysis has been carried out. The main source of systematic errors is found to be the uncertainty on the subtraction of the proton dissociative DVCS background. This leads to an uncertainty on the final cross section of between $8 \%$ and $14 \%$ in the highest $|t|$ bin. The total systematic error is typically $20 \%$. 

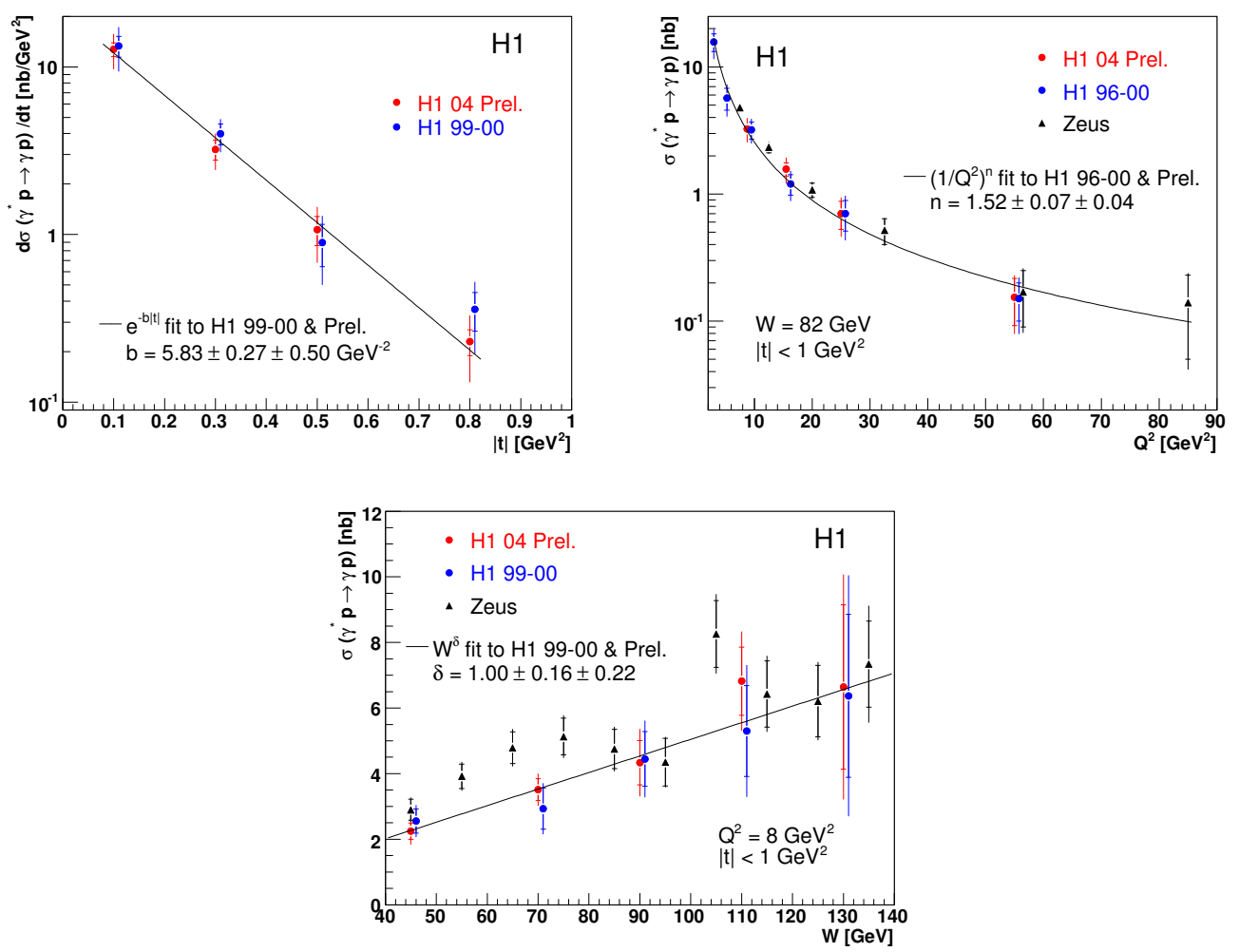

Figure 2: The $\gamma^{*} p \rightarrow \gamma p$ cross section differential in (a) $|t|$ at $Q^{2}=8 \mathrm{GeV}^{2}$ and $W=82 \mathrm{GeV}$, (b) $Q^{2}$ at $W=82 \mathrm{GeV}$ and (c) $W$ at $Q^{2}=8 \mathrm{GeV}^{2}$. The inner error bars show the statistical error and the outer error bars show the statistical and systematic errors added in quadrature. The data are compared to fits on the combined $\mathrm{H} 1$ data points of $\exp (-b|t|),\left(1 / Q^{2}\right)^{n}$ and $W^{\delta}$, respectively.

\subsection{Results}

The H1 2004 preliminary results are shown in the kinematic range $6.5<Q^{2}<80 \mathrm{GeV}^{2}$, $30<W<80 \mathrm{GeV}$ and $|t|<1 \mathrm{GeV}^{2}$, compared to the previously published H1 [16] and ZEUS measurements [17]. The $\gamma^{*} p \rightarrow \gamma p$ cross section differential in $t$ at $Q^{2}=8 \mathrm{GeV}^{2}$ and $W=82 \mathrm{GeV}$ is shown in Figure 2 (a). The H1 2004 preliminary measurement is in good agreement with the published $\mathrm{H} 1$ measurement. A combined fit to the two $\mathrm{H} 1$ measurements of the form $\exp (-b|t|)$ yields the value $b=5.83 \pm 0.27$ (stat.) \pm 0.05 (sys.) $\mathrm{GeV}^{-2}$. The $\gamma^{*} p \rightarrow \gamma p$ cross section as a function of $Q^{2}$ at $W=82 \mathrm{GeV}$ and $W$ at $Q^{2}=8 \mathrm{GeV}^{2}$ are shown in figure 2 (b) and (c) respectively. Once again there is good agreement between the two $\mathrm{H} 1$ analyses and also generally good agreement with the ZEUS published analysis. However, the ZEUS data seems to sit a little higher than the $\mathrm{H} 1$ data sets at $W \sim 70 \mathrm{GeV}$. A fit to the $Q^{2}$ dependence of two H1 results of the form $\left(1 / Q^{2}\right)^{n}$ gives $n=1.52 \pm 0.07$ (stat.) \pm 0.04 (sys.), while a fit to the $W$ dependence of the form $W^{\delta}$ gives $\delta=1.00 \pm 0.16$ (stat.) \pm 0.22 (sys.). The steep rise with $W$ indicates the hard scale present and is even somewhat harder than that seen for diffractive $J / \psi$ production $[18,19]$.

Figure 3 shows the $\gamma^{*} p \rightarrow \gamma p$ cross section as a function of (a) $Q^{2}$ and (b) $W$ compared to the NLO QCD prediction from Freund and McDermott. Two different parametrisations for the proton PDFs, MRST 2001 and CTEQ6, are used as inputs for the GPDs in the DGLAP region. 

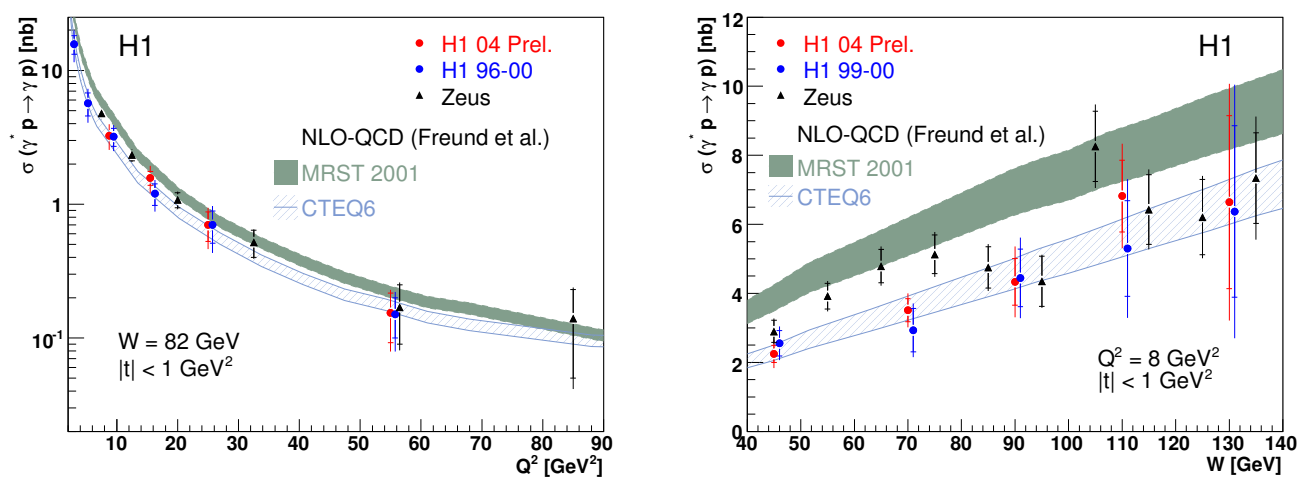

Figure 3: The $\gamma^{*} p \rightarrow \gamma p$ cross section differential in (a) $Q^{2}$ at $W=82 \mathrm{GeV}$ and (b) $W$ at $Q^{2}=8 \mathrm{GeV}^{2}$. The H1 2004 preliminary data are compared to the previous H1 and ZEUS measurements and a NLO QCD prediction from Freund and McDermott using the CTEQ6 and MRST2001 PDFs as inputs.
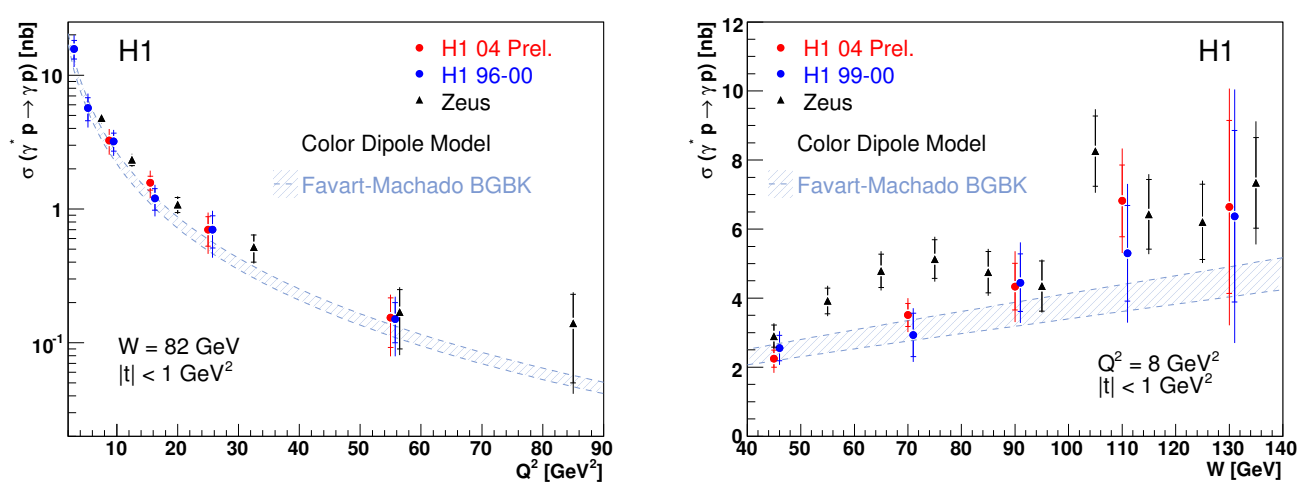

Figure 4: The $\gamma^{*} p \rightarrow \gamma p$ cross section differential in (a) $Q^{2}$ at $W=82 \mathrm{GeV}$ and (b) $W$ at $Q^{2}=8 \mathrm{GeV}^{2}$. The H1 2004 preliminary data are compared to the previous H1 and ZEUS measurements and a colour dipole model prediction from Favart and Machado.

The uncertainty on the normalisation of the prediction, shown by the error band, is reduced by the improved statistical precision of the $t$ slope parameter $b$ from this analysis. There is a good description of the data by the NLO QCD prediction and sensitivity of the model to the input PDFs, reflecting the relative sizes of the input singlet and gluon distributions, is seen. This good agreement between model prediction and data is observed without any intrinsic skewedness in the NLO QCD model of the GPDs.

Figure 4 shows the $\gamma^{*} p \rightarrow \gamma p$ cross sections as a function of (a) $Q^{2}$ and (b) $W$ compared to a colour dipole model prediction from Favart and Machado. The model gives a reasonable description of the data, however the predicted $W$ dependence is somewhat softer than that observed in the data. Again, the error band on the prediction corresponds to the normalisation error from the uncertainty on the $t$ slope measurement. 


\section{Diffractive High $t$ Photons}

\subsection{Data Analysis}

The preliminary measurement of diffractive high $t$ photon production is based on an integrated luminosity of $47.6 \mathrm{pb}^{-1}$ collected by the $\mathrm{H} 1$ detector during the 1999-2000 running period. During this time HERA collided positrons with an energy of $27.6 \mathrm{GeV}$ with protons of energy $920 \mathrm{GeV}$. More details of the analysis may be found in [2].

Photoproduction events are selected by tagging the scattered positron in the electron tagger of the luminosity system. This restricts the virtuality of the photon to $Q^{2}<0.01 \mathrm{GeV}^{2}$ and the photon-proton centre of mass energy to $175<W<247 \mathrm{GeV}$. The scattered photon is identified in the $\mathrm{SpaCal}$ as an electromagnetic cluster of energy $E>8 \mathrm{GeV}$ with a low cluster radius, no energy within the hadronic region of the SpaCal and with no track associated with the cluster. Hadronic final state objects are defined, using a combination of tracking and calorimetric information, by an algorithm that avoids the double counting of tracks and calorimeter clusters.

Diffractive events are selected by requiring $y_{\mathbb{P}}<0.018$, where

$$
y_{\mathbb{P}}=\frac{p \cdot(q-X)}{q \cdot p} \simeq \frac{\sum_{Y}\left(E-p_{z}\right)}{2 E_{\gamma}} .
$$

Here, $p$ and $q$ are the four-vectors of the incoming proton and photon, respectively, and $\mathrm{X}$ is the four-vector of the final state $X$ system - in this process the final state photon. The quantity is calculated experimentally by summing over all of the hadronic final state objects (ie. the final state, excluding the scattered positron and photon) and dividing by twice the energy of the incoming photon energy $E_{\gamma}$. This method has the experimental advantage that particles lost along the forward beam pipe do not contribute significantly to the numerator. As $y_{\mathbb{P}} \simeq \exp (-\Delta \eta)$, this cut ensures a large rapidity gap between the photon and dissociated proton system. However, no proton dissociated system is required to be observed within the $\mathrm{H} 1$ detector. To ensure efficient background selection, a minimum rapidity separation of $\Delta \eta>2$ is required between the photon and the edge of the proton system. This condition is corrected for in the final cross sections. In addition, the kinematic variable $x_{\mathbb{P}}$ is also defined as

$$
x_{\mathbb{P}}=\frac{q \cdot(p-Y)}{q \cdot p} \simeq \frac{\left(E+p_{z}\right)_{\gamma}}{2 E_{p}}
$$

where $Y$ is the four-vector of the proton dissociative system, $\left(E+p_{z}\right)_{\gamma}$ is the $E+p_{z}$ of the photon candidate and $E_{p}$ is the energy of the incident proton.

\subsection{Monte Carlo Simulations and Data Corrections}

The HERWIG 6.1 Monte Carlo generator [20] was used to correct the data for detector acceptance and bin migration effects and to make model comparisons. Details of the implementation of the diffractive high $t$ photon process may be found in [21]. The calculation has been performed within the leading logarithmic approximation (LLA) of BFKL and includes contributions from both real and virtual incoming photons [22, 23]. At leading logarithmic accuracy, the strong coupling constant $\alpha_{s}$ is a fixed parameter. This means that there are two independent free parameters in the calculation: the value of $\alpha_{s}$ in the pre-factor of the cross section (which enters to the fourth 

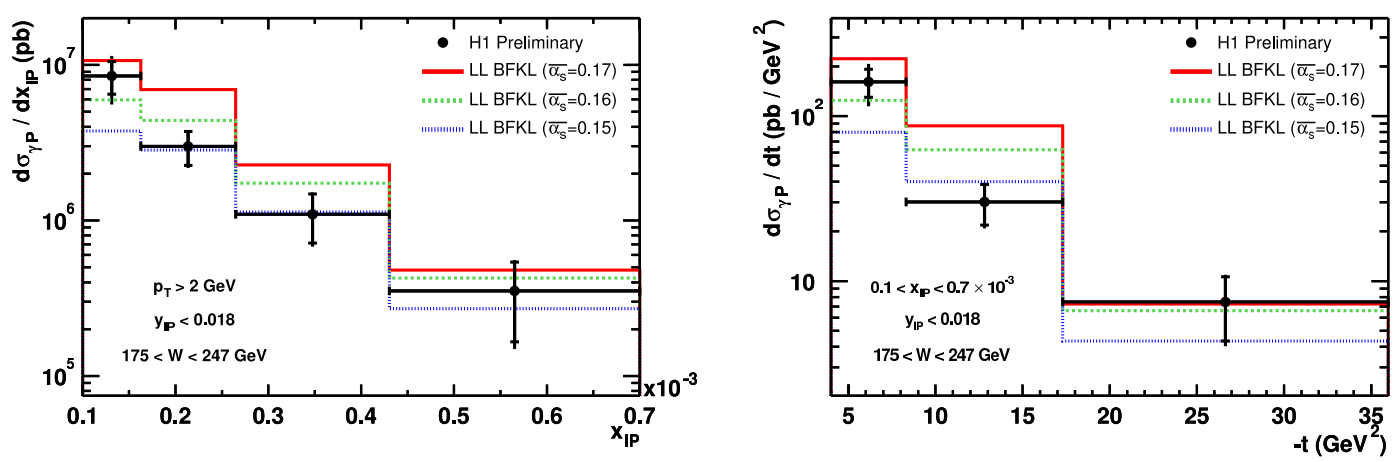

Figure 5: The $\gamma p$ cross section differential in (a) $x_{\mathbb{P}}$ and (b) $|t|$, in the kinematic range $p_{t(\gamma)}>2 \mathrm{GeV}$, $175<W<247 \mathrm{GeV}, 1 \times 10^{-4}<x_{\mathbb{P}}<7 \times 10^{-4}$ and $y_{\mathbb{P}}<0.018$. The inner error bars show the statistical error and the outer error bars show the statistical and systematic errors added in quadrature. The dotted line shows the prediction of the leading logarithmic approximation of BFKL as implemented in HERWIG, for the choice of fixed $\overline{\alpha_{s}}=0.15$ in the prefactor and pomeron intercept, as described in the text. The dashed line and solid line show the same calculation, for $\overline{\alpha_{s}}=0.16$ and $\overline{\alpha_{s}}=0.17$ respectively.

power) and the value of $\alpha_{s}$ in the pomeron intercept $1+\omega_{0}$, where $\omega_{0}=\left(3 \alpha_{s} / \pi\right) 4 \ln 2$ in the LLA. In all that follows, the two values of $\alpha_{s}$ are chosen to be equal $\left(\alpha_{s}\right.$ will henceforth be referred to as $\left.\overline{\alpha_{s}}\right)$. Note, however, that this is an arbitrary choice. In particular, to the accuracy of the present calculations

$$
\frac{\mathrm{d} \sigma}{\mathrm{d} x_{\mathbb{P}}} \sim \frac{1}{W^{2}}\left(\frac{1}{x_{\mathbb{P}}}\right)^{2 \omega_{0}+2}
$$

so the slope of the $x_{\mathbb{P}}$ distribution is affected only by the choice of $\overline{\alpha_{s}}$ in the pomeron intercept.

A HERWIG sample with $\overline{\alpha_{s}}=0.17$ was passed through a full simulation of the H1 detector and is subject to the same reconstruction and analysis chain as the data. This sample was found to give a good description of the data for all kinematic distributions considered. To investigate the model dependence of the detector correction procedure, the MC sample was reweighted in $x_{\mathbb{P}}$, $p_{T(\gamma)}$ and the mass of the dissociated proton system, $M_{Y}$. The model dependence is included in the systematic errors on the final cross sections. The largest systematic error is due to the error in the noise subtracted from the LAr calorimeter.

The background from inclusive diffractive photoproduction events was simulated using the PHOJET Monte Carlo generator [24]. A small contribution from this background of less than 9\% was found and subtracted on a bin-by-bin basis. To investigate the background from diffractive $\omega$ production, where the $\omega$ decays through the $\pi^{0} \gamma$ or $\pi^{+} \pi^{-} \pi^{0}$ channels, a sample was generated using the DIFFVM Monte Carlo generator [14]. The contribution was found to be negligible.

\subsection{Results}

The $\gamma p$ cross section differential in $x_{\mathbb{P}}$, in the range $175<W<247 \mathrm{GeV}, p_{t(\gamma)}>2 \mathrm{GeV}$ and $y_{\mathbb{P}}<0.018$, is shown in figure 5 (a). The cross section is defined at the level of stable hadrons. Also shown are the LLA BFKL predictions from the HERWIG MC. The data are sensitive to the choice of the $\overline{\alpha_{s}}$ parameter in the prediction in two ways - the first is the slope of the $x_{\mathbb{P}}$ distribution which, as equation 4.3 shows, is related to the BFKL pomeron intercept. The cross section clearly rises 
steeply as $x_{\mathbb{P}}$ becomes small - the classic signature of a diffractive process. Within the errors, it is difficult to distinguish between the slopes of the 3 choices of $\overline{\alpha_{s}}$, although perhaps $\overline{\alpha_{s}}=0.15$ leads to a rise with $1 / x_{\mathbb{P}}$ that is too shallow when compared to the data. The second point of interest is the normalisation of the cross section predictions. Here, a choice of $\overline{\alpha_{s}}=0.17$ produces a cross section with a normalisation which is too large compared to that observed in the data. It should be noted, however, that there is some normalisation uncertainty within the LLA, even given a choice of $\overline{\alpha_{s}}$. It may therefore be possible to fix both $\overline{\alpha_{s}}$ values at 0.17 and obtain a good description of the data in both normalisation and shape. This is in comparison to the value $\overline{\alpha_{s}}=0.18$ as used for recent $\mathrm{H} 1$ measurements [26, 25].

The $\gamma p$ cross section differential in the squared four-momentum transfer between proton and the incoming photon $t$ (where in photoproduction $-t \sim p_{t(\gamma)}^{2}$ ), is shown in figure 5 (b) in the range $175<W<247 \mathrm{GeV}, 1 \times 10^{-4}<x_{\mathbb{P}}<7 \times 10^{-4}$ and $y_{\mathbb{P}}<0.018$. In this case, the agreement in the shape of the cross section between the HERWIG predictions and the data is perhaps more questionable, for all values of $\overline{\alpha_{s}}$ chosen here.

\section{Conclusions}

A new preliminary $\mathrm{H} 1$ measurement of the DVCS cross section is presented alongside the preliminary $\mathrm{H} 1$ measurement of diffractive high $t$ photon production. The DVCS results, based on data collected in the year 2004 during the HERA II running period, are measured in the kinematic range $6.5<Q^{2}<80 \mathrm{GeV}^{2}, 30<W<80 \mathrm{GeV}$ and $|t|<1 \mathrm{GeV}^{2}$ as a function of $t, Q^{2}$ and $W$. The new results are in good agreement with the previously published $\mathrm{H} 1$ results and are compatible with the ZEUS measurement. Fits to the combined H1 results of the form $\exp (-b|t|),\left(1 / Q^{2}\right)^{n}$ and $W^{\delta}$ are able to describe the $t, Q^{2}$ and $W$ cross sections, respectively. The increased statistical precision of the $t$ slope improves the constraint of the normalisation of the theoretical predictions. The data are described by NLO QCD and colour dipole model predictions in both shape and normalisation.

Diffractive high $t$ photon production, the process $e \gamma p \rightarrow e \gamma Y$ where the photon carries a large transverse momentum and is well separated from the proton dissociative system $Y$, has been studied. Cross sections have been measured differentially in the appropriate energy variable $x_{\mathbb{P}}$ and the squared four-momentum transfer at the proton vertex, $t$, in the kinematic range $p_{t(\gamma)}>2 \mathrm{GeV}$, $175<W<247 \mathrm{GeV}, 1 \times 10^{-4}<x_{\mathbb{P}}<7 \times 10^{-4}$ and $y_{\mathbb{P}}<0.018$. The cross sections are found to be in reasonable agreement in both normalisation and shape with the predictions of the leading logarithmic approximation of BFKL. In particular, the cross section is found to rise very steeply with decreasing $x_{\mathbb{P}}$.

\section{References}

[1] H1 Collaboration, paper 9 ICHEP06, H1prelim-06-012.

[2] H1 Collaboration, paper 183 ICHEP04, H1 prelim-03-012.

[3] I. Abt et al. [H1 Collaboration], Nucl. Instrum. Meth. A 386 (1997) 310.

[4] A. Freund and M. F. McDermott, Phys. Rev. D 65 (2002) 091901 [arXiv:hep-ph/0106124].

[5] A. Freund and M. McDermott, Eur. Phys. J. C 23 (2002) 651 [arXiv:hep-ph/0111472]. 
[6] A. Freund, M. McDermott and M. Strikman, Phys. Rev. D 67 (2003) 036001 [arXiv:hep-ph/0208160].

[7] L. Favart and M. V. T. Machado, Eur. Phys. J. C 29 (2003) 365 [arXiv:hep-ph/0302079].

[8] L. Favart and M. V. T. Machado, Eur. Phys. J. C 34 (2004) 429 [arXiv:hep-ph/0402018].

[9] K. Golec-Biernat and M. Wusthoff, Phys. Rev. D 60 (1999) 114023 [arXiv:hep-ph/9903358].

[10] J. Bartels, K. Golec-Biernat and H. Kowalski, Acta Phys. Polon. B 33 (2002) 2853 [arXiv:hep-ph/0207031].

[11] E. Perez, L. Schoeffel and L. Favart, arXiv:hep-ph/0411389.

[12] T. Carli, A. Courau, S. Kermiche and P. Kessler, "Quasireal QED Compton Monte Carlo," In *Hamburg 1991, Proceedings, Physics at HERA, vol. 2*902-915.

[13] A. Mucke, R. Engel, J. P. Rachen, R. J. Protheroe and T. Stanev, Comput. Phys. Commun. 124 (2000) 290 [arXiv:astro-ph/9903478].

[14] B. List and A. Mastroberardino, "DIFFVM: A Monte Carlo generator for diffractive processes in e p scattering," Prepared for Workshop on Monte Carlo Generators for HERA Physics (Plenary Starting Meeting), Hamburg, Germany, 27-30 Apr 1998.

[15] T. Abe, Comput. Phys. Commun. 136 (2001) 126 [arXiv:hep-ph/0012029].

[16] A. Aktas et al. [H1 Collaboration], Eur. Phys. J. C 44 (2005) 1 [arXiv:hep-ex/0505061].

[17] S. Chekanov et al. [ZEUS Collaboration], Phys. Lett. B 573 (2003) 46 [arXiv:hep-ex/0305028].

[18] C. Adloff et al. [H1 Collaboration], Eur. Phys. J. C 10 (1999) 373 [arXiv:hep-ex/9903008].

[19] S. Chekanov et al. [ZEUS Collaboration], Eur. Phys. J. C 24 (2002) 345 [arXiv:hep-ex/0201043].

[20] G. Marchesini, B. R. Webber, G. Abbiendi, I. G. Knowles, M. H. Seymour and L. Stanco, Comput. Phys. Commun. 67 (1992) 465.

[21] B. E. Cox and J. R. Forshaw, J. Phys. G 26 (2000) 702 [arXiv:hep-ph/9912486].

[22] D. Y. Ivanov and M. Wusthoff, Eur. Phys. J. C 8 (1999) 107 [arXiv:hep-ph/9808455].

[23] N. G. Evanson and J. R. Forshaw, Phys. Rev. D 60 (1999) 034016 [arXiv:hep-ph/9902481].

[24] F. W. Bopp, R. Engel and J. Ranft, arXiv:hep-ph/9803437.

[25] A. Aktas et al. [H1 Collaboration], Phys. Lett. B 568 (2003) 205 [arXiv:hep-ex/0306013].

[26] C. Adloff et al. [H1 Collaboration], Eur. Phys. J. C 24 (2002) 517 [arXiv:hep-ex/0203011]. 